\title{
Hybrid Photoactive Assemblies: Electron Injection from Host-Guest Complexes into Semiconductor Nanoparticles
}

\author{
Cynthia Pagba, Giovanni Zordan, Elena Galoppini, Kurt Deshayes, \\ Eugene L. Piatnitski, Sarmimala Hore, Piotr Piotrowiak
}

\section{Supporting Materials}

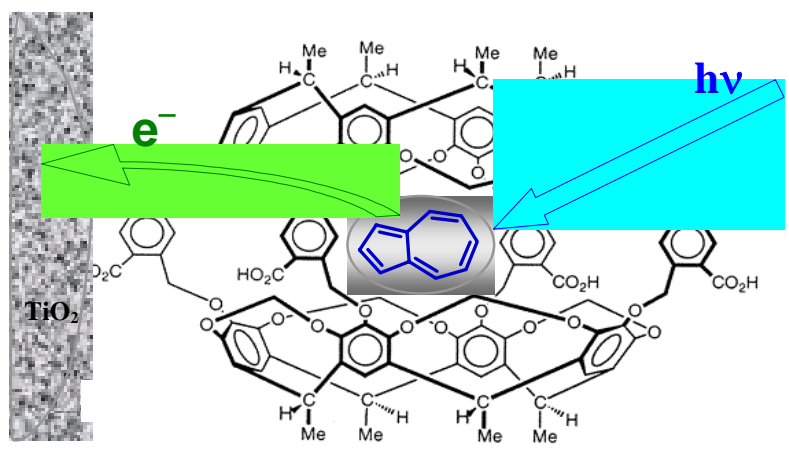

$\mathrm{TiO}_{2}$ preparation: Slightly modified procedure described by Hartland et. al., J. Phys. Chem. B 1999, 103: 9104-9111, was followed. A freshly prepared solution of $3 \mathrm{~mL}$ of titanium (IV) isopropoxide (Acros, 98+\%) in $60 \mathrm{~mL}$ of isopropanol was added slowly ( $1 \mathrm{drop} / \mathrm{sec}$ ) to 300 $\mathrm{mL}$ of oxygen-free water $(\mathrm{pH}=1, \mathrm{HCl})$ at $0^{\circ} \mathrm{C}$ under nitrogen and vigorous stirring. The resulting solution was stirred at $0^{\circ} \mathrm{C}$ for $3 \mathrm{~h}$ with continued nitrogen bubbling. It was then left overnight (with continuous stirring, without nitrogen) at room temperature. The solvent was removed by rotary evaporation. The average particle size was determined by light scattering measurements (Wyatt Technology Dawn EOS, with Wyatt QELS detector).

\section{UV-Vis absorption spectra:}

UV-Vis absorption spectra of $1 \times 10^{-4} \mathrm{M}$ 1-carboxyazulene bound to $\mathrm{TiO}_{2}$ (blue) and $2 \times 10^{-4} \mathrm{M}$ azulene encapsulated within the hemicarcerand collected on a Carry 500 UV-Vis-NIR spectrometer. Note that the $\mathrm{S}_{0}-\mathrm{S}_{2}$ absorption of carboxyazulene on $\mathrm{TiO}_{2}$ extends to nearly $400 \quad \mathrm{~nm} \quad$ allowing ultrafast

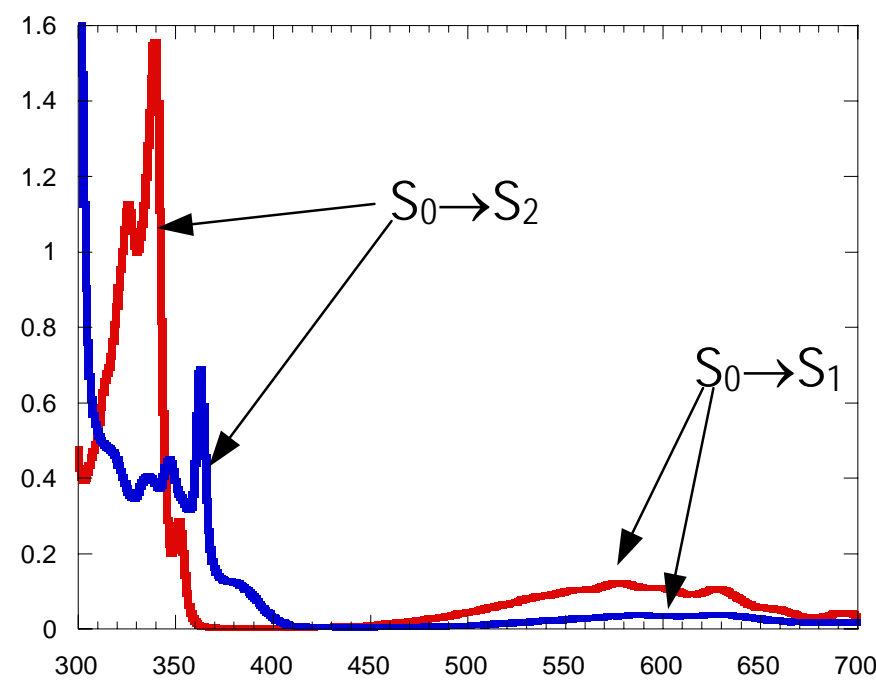

Figure S1. UV-Vis absorption of 1-carboxyazulene on $\mathrm{TiO}_{2}$ (blue) and encapsulated azulene in solution (red). 
spectroscopy measurements (the currently

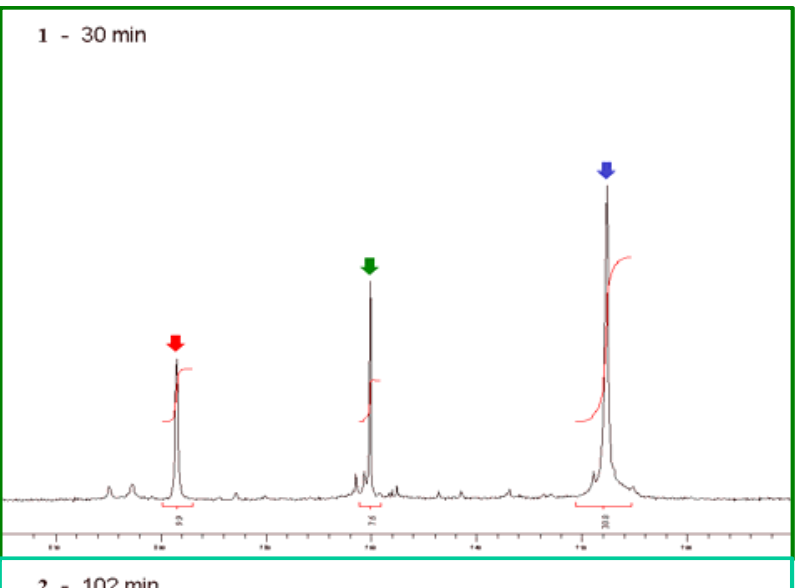
experiments.

${ }^{1}$ H-NMR spectra: NMR spectra were recorded on 500 and $400 \mathrm{MHz}$ Varian Infinity series spectrometers. The encapsulation reaction was carried out in a NMR tube by adding an excess of solid azulene to a $1 \mathrm{mg} / \mathrm{ml}$ solution of the octacarboxyhemicarcerand in $\mathrm{D}_{2} \mathrm{O}$ at $\mathrm{pH}=$ 8.0. The encapsulation process is accompanied by major changes in chemical shifts of both the guest molecule and the hemicarcerand host. Here we focus on the evolution of the aromatic signals of the hemicarceplex as it encapsulates the azulene. Specifically, protons $a$ shift from $7.15 \mathrm{ppm}$ to $7.43 \mathrm{ppm}$ (blue arrow), protons $b$ from $7.97 \mathrm{ppm}$ to $7.58 \mathrm{ppm}$ (red) and protons $c$ from $7.60 \mathrm{ppm}$ to $7.47 \mathrm{ppm}$ (green). In addition, the appearance of the doublet assigned to the 1,3 protons of azulene

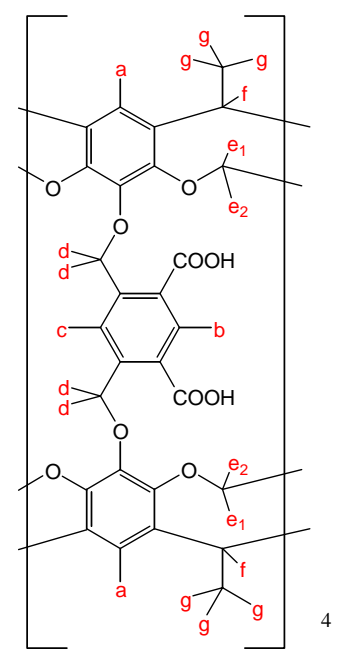

$2-102 \mathrm{~min}$
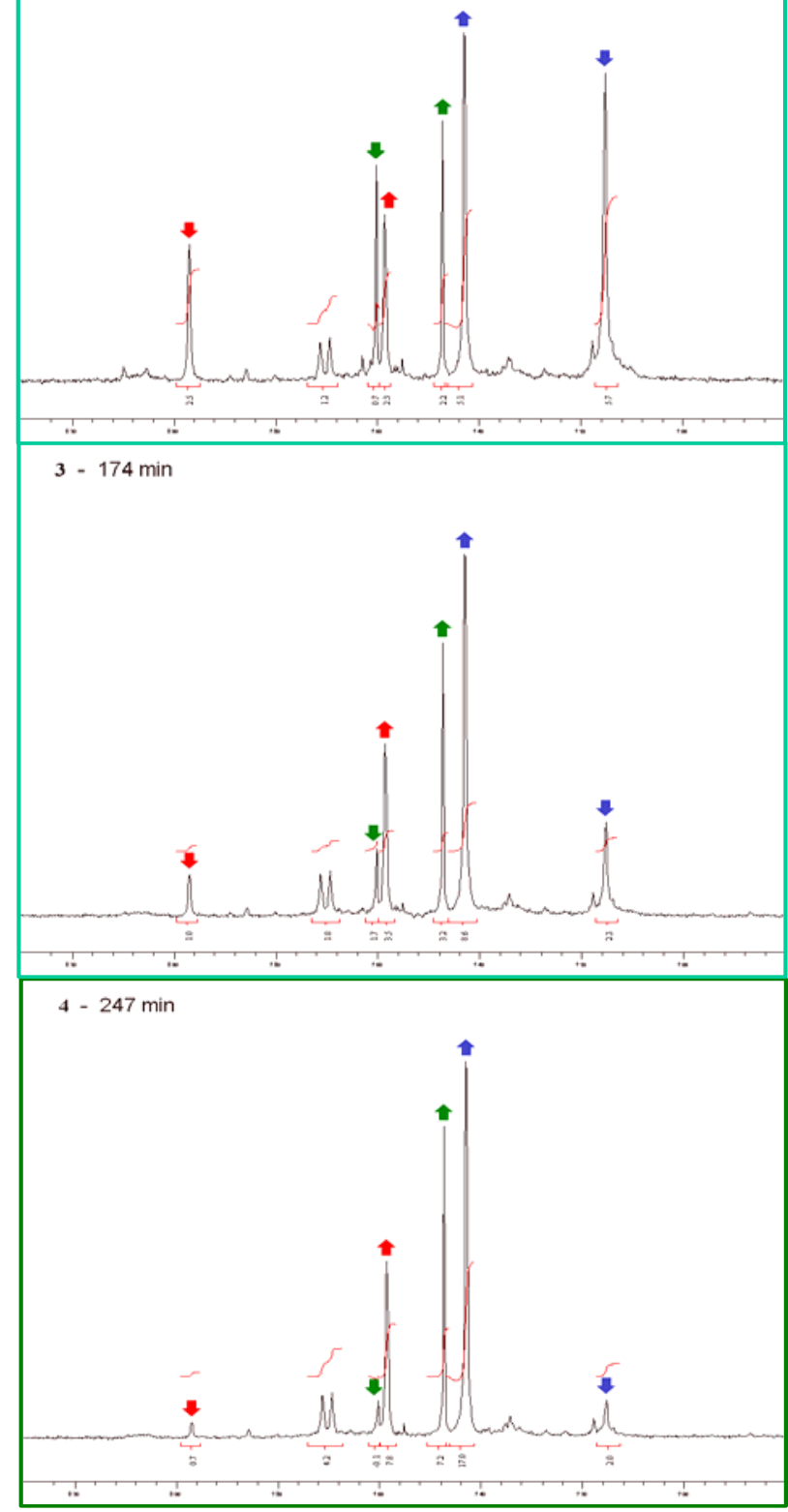

Figure S2. Encapsulation of azulene by the octacarboxyhemicarcerand monitored by ${ }^{1} \mathrm{H}-\mathrm{NMR}$. 
is seen at $7.70 \mathrm{ppm}$ (normal chemical shift in $\mathrm{CDCl}_{3}$ at $8.38 \mathrm{ppm}$ ). The integration confirms that a 1:1 host guest complex has been formed. The downfield shift of protons $a$ of the hemicarcerand host supports results of the Spartan AM1 optimization, which places the plane of azulene parallel to the principal axis of the host.

\section{Nanosecond transient absorption data:}

The nanosecond transient absorption experiments were performed on thoroughly degassed solutions (suspensions) of the host-guest encapsulated complex $\mathbf{1}$ bound to $\mathrm{TiO}_{2}$ nanoparticles. Samples were excited at $355 \mathrm{~nm}\left(3^{\text {rd }}\right.$ harmonic of a Q-switched Nd:YAG laser, Brilliant B, Quantel, approximate pulse energy $20 \mathrm{~mJ}$ ). Xenon flashlamp was used as the source of analyzing light, which after passing the sample was dispersed using an M257 Oriel monochromator and focused onto a Si photodiode. The signal was digitized with a Tektronix SDC 1000 oscilloscope. 16 scans of I and $\mathrm{I}_{0}$ were taken at each wavelength. The decays of the characteristic broad NIR absorption band of the radical cation of the encapsulated azulene (see Electronic Absorption Spectra of Radical Ions, Tadamasa Shida, Elsevier, 1988) are shown on the right. The sharp negative feature present at the beginning of each transient is the result of light scattering by the colloidal $\mathrm{TiO}_{2}$.
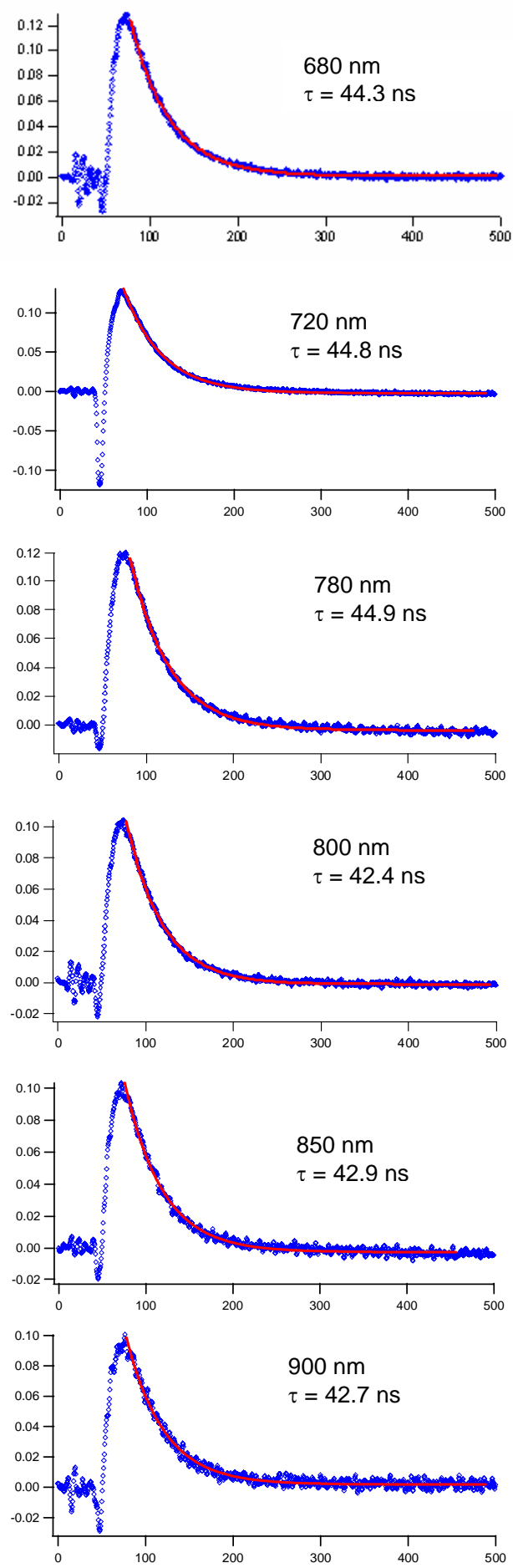

Figure S3. Decay of the encapsulated radical cation of azulene following electron injection into $\mathrm{TiO}_{\text {, monitored in the NIR. }}$ 